

Asian-Aust. J. Anim. Sci.

Vol. 25, No. 3 : 435 - 446

March 2012

www.ajas.info

http://dx.doi.org/10.5713/ajas.2011.11286

\title{
Flavor Characteristics of Hanwoo Beef in Comparison with Other Korean Foods
}

\author{
Hoa Van Ba, Kyeong-Seon Ryu and Hwang Inho* \\ Department of Animal Science and Institute of Rare Earth for Biological Application, \\ Chonbuk National University, Jeonju, 561-756, Korea
}

\begin{abstract}
The present study identified volatile flavor components of Hanwoo longissimus muscle and other Korean foods (Doenjang, Chungukjang, sesame oil) and their traits were compared in relation with flavor precursors that include fatty acids and protein degradation products. Hanwoo longissimus muscle was purchased from a commercial abattoir while the other foods were sampled from three separate households. The results showed totals of 68 (9.94 $\mu \mathrm{g} / \mathrm{g}), 60(15.75 \mu \mathrm{g} / \mathrm{g}), 49(107.61 \mu \mathrm{g} / \mathrm{ml}) \mathrm{and} 50$ (7.20 $\mu \mathrm{g} / \mathrm{g}$ ) volatile components for Doenjang, Chungukjang, sesame oil and Hanwoo beef longissimus, respectively ( $\mathrm{p}<0.05)$. Aldehydes were the most predominant components in beef, but alcohols, acids and esters, and pyrazines are probably the major contributors to the flavor characteristics of other foods. SDS-PAGE revealed that beef longissimus muscle and Doenjang showed higher protein degradation than other foods which could be likely related to chiller ageing and ripening process. The total polyunsaturated fatty acids were approximately 50, 60, 41 and 5\% for Doenjang, Chungukjang, sesame oil and beef longissimus muscle, respectively. Based on the mechanism(s) of generation of the volatile compounds and the chemical composition of each food sample, differences and traits of volatile flavor components among the four food types are likely due to fatty acid profiles, proteolytic activity and processing conditions. Aroma intense compounds like pyrazines and sulfur-containing compounds were limited in cooked beef in the current experimental condition (i.e., relatively low heating temperature). This suggests that higher heating temperature as in the case of roasting is needed for the generation of high aroma notes in meat. Furthermore, proteolytic activity and stability of fatty acids during ageing have a great influence on the generation of flavor components in cooked beef. (Key Words : Chungukjang, Doenjang, Hanwoo Beef, Sesame Oil, Volatile Flavor Component)
\end{abstract}

\section{INTRODUCTION}

Hanwoo beef is the most preferred among the meats consumed by Korean consumers due to higher marbling, flavor, tenderness, and juiciness compared with beef from other breeds or imported beef (Kim et al., 1993; Kim and Lee, 2003; Hwang et al., 2010). A great amount of information on the objective and subjective quality characteristics of Hanwoo beef have been documented over the last decades (Kim and Lee, 2003; Panjono et al., 2009; Hwang et al., 2010). Studies indicated that meat flavor together with tenderness is the most important criterion of acceptability and palatability of beef that affects consumers' purchasing decisions (Savell et al., 1987; Robbins et al., 2003). However, we are not aware of any accessible

\footnotetext{
* Corresponding Author : Inho Hwang. Tel : +82-63-270-2605, Fax : +82-63-270-2605, E-mail : inho.hwang@jbnu.ac.kr Submitted Aug. 19, 2011; Accepted Oct. 25, 2011; Revised Nov. 3, 2011
}

information on volatile flavor components of Hanwoo beef and their comparison with other Korean foods.

Sesame oil and fermented soybean products are the traditional foods which are widely consumed in many Asian countries including Korea, China, Taiwan, Thailand and Vietnam. Doenjang and Chungukjang, the popular fermented soybean products, and sesame oil are likely the prime aroma source of several foods in the daily meal of Korean people. Sesame seeds are the important oil seeds which are commonly produced in several countries in Asia and Africa like China, India and Nigeria. Roasting and pressing sesame seeds are two main processes that are currently used in the production of sesame oil (Chung and Choe, 2001). For a long time, sesame oil is used as natural salad oil and cooking oil. Its total unsaturated fatty acid content amounts to $85 \%$ (Sonntage, 1981). Doenjang and Chungukjang are manufactured through the natural fermentation of cooked soybean in the presence of fermenting agents at the suitable conditions. The total 
unsaturated fatty acid in fermented soybean is about $80 \%$ and the amounts of these fatty acids tend to increase throughout the fermentation process (Park et al., 2010).

Flavor characteristics of foods are directly detected by the nose (i.e., olfactory receptors) before and during chewing. It has been well documented that volatile flavor components of food flavor are driven by four main sources including the Maillard reactions of amino acids with reducing sugars, thermal degradation of fatty acids, vitamin degradation and interaction of the intermediates of Maillard reaction with lipid-degradation products during cooking (Mottram, 1994; Macleod, 1994). Several volatile flavor compounds contributing to the distinct flavors of these food types have been identified in previous works such as 2ethyl-6-methylpyrazine, 2-ethyl-5- methylpyrazine, 4,5dimethylisothiazole, 4,5-dimethylthiazole, 2,6-diethylpyrazine, 2-ethyl-2,5-dimethylpyrazine and 1-(2-pyridinyl)ethanone which are the principle contributors of sesame oil flavor (Shimoda et al., 1996); and 2,3-butanediol, acetic acid, benzene acetaldehyde and pyrazine 2,6-dimethyl, maltol, pyrazines, 1-octen-3-ol which were described as the major odor - active compounds in fermented soybean (Lee and Ahn, 2009; Zhao et al., 2011); Dimethyl sulphide, 2-butanone, ethyl acetate, 2- and 3-methylbutanal, 2-heptanone, dimethyl trisulphide and nonanal were detected as key flavor compounds of cooked Irish Angus beef while methional, 2,4-nonadienal and bezothiazole were known to impart meaty, oily notes in cooked Belgian Blue, Limousin and Aberdeen Angus beefs (Machiel et al., 2003).

Both the composition of raw material and processing methods has direct influence on the characteristic flavor characteristics of these food types. The types and concentrations of free amino acids/peptides, fatty acids, reducing sugars in foods influence flavor traits as a consequence of their participation in the reactions, oxidation and degradation processes during heating to form flavor components (Macleod, 1994; Mottram, 1994; Elmore et al., 2002). In addition, moisture content, $\mathrm{pH}$, and heating conditions affect types and concentrations of volatile flavor components (Shi and Ho, 1994; Madruga and Mottram, 1995; Ames et al., 2001).

In general, Korean consumers could recognize flavor notes of fermented Doenjang and Chungukjang, and sesame oil while we are unaware whether Korean beef consumers could remember flavor traits of Hanwoo beef. Flavor note is an important quality trait of food including beef, but characterization of volatile flavor components of Hanwoo beef in comparison with traditional local food has not been done. The current study was conducted to evaluate and compare the volatile flavor components and their related flavor precursors of Hanwoo longissimus muscle, Doenjang, Chungukjang and sesame oil.

\section{MATERIALS AND METHODS}

\section{Materials}

Fermented soybean including traditionally made Doenjang and Chungukjang, and sesame oil were sampled from three separate households in Jeonju, South Korea. Longissimus dorsi beef muscle from Hanwoo was purchased from a commercial abattoir, vacuum packaged and aged for $7 \mathrm{~d}$ at $4{ }^{\circ} \mathrm{C}$ then was frozen at $-20^{\circ} \mathrm{C}$ until analysis. The experiment was carried out three times $(n=3)$.

An internal standard compound, 2-methyl-3-methanone, $n$-alkane $\left(\mathrm{C}_{8}-\mathrm{C}_{20}\right)$ and authentic compounds including acetaldehyde, trans-2-decenal, 2-methylpropanal, 2methylbutanal, 3-methylbutanal, pentanal, hexanal, heptanal, (E),2-heptenal, 2-methylthiazole, benzaldehyde, octanal, benzeneacetaldehyde, nonanal, decanal, (E,E), 2,4decadienal, ethanol, 1-propanol, 2-furanmethanol, 1-hexanol, 1-octen-3-ol, fufural, tetradecanal, 1-octanol, acetone, 2-propanone, 2,3-butanedione, 2-butanone, 2-heptanone, 3-octanone, dimethyldisulfide, pyrazine, methylpyrazine, 2,3-dimethylpyrazine etc. were purchased from SigmaAldrich (St. Louis, MO, USA).

\section{Fatty acid extraction and analysis}

Extraction of fatty acids in Doenjang and Chungukjang were done following the method described by Park et al. (2010) with minor modifications. Five-hundred (500) mg of each freeze-dried Doenjang and Chungukjang were placed into $20 \mathrm{ml}$ headspace vial with silicone-lined cap then $3 \mathrm{ml}$ of $0.5 \mathrm{M}$ methanolic $\mathrm{NaOH}$ solution was added and sealed with scrimp cap. The vial was kept in the heating block set at $80^{\circ} \mathrm{C}$ for $20 \mathrm{~min}$ then cooled at room temperature. Thereafter, $3 \mathrm{ml}$ of $14 \%$ boron trifluoride in methanol (DBH, DBH Lab. Supplies, Poole, England) was added, sealed and placed in the heating block set at $80^{\circ} \mathrm{C}$ for 20 min. Immediately after cooling the vial, $3 \mathrm{ml}$ distilled water and $3 \mathrm{ml}$ hexane were added. The vial was then centrifuged at $1,000 \times \mathrm{g}$ for $5 \mathrm{~min}$ to accelerate phase separation. The supernatant hexane layer was transferred to a new vial containing $1 \mathrm{~g}$ of anhydrous sodium sulfate to remove water. One (1.0) $\mathrm{ml}$ concentrated upper solution was transferred to GLC auto-sampler vials for fatty acid analysis.

Fatty acids in sesame oil were extracted following the method described by Lee et al. (1998) with suitable modifications. Briefly, $0.5 \mathrm{ml}$ of sesame oil was placed into a $20 \mathrm{ml}$ headspace vial with silicone-lined cap and $3 \mathrm{ml}$ of $0.5 \mathrm{M}$ methanolic $\mathrm{NaOH}$ solution was added and sealed with scrimp cap. The vial was placed on the heating block set at $80^{\circ} \mathrm{C}$ and kept for $10 \mathrm{~min}$. After cooling the vial at room temperature, $3 \mathrm{ml}$ of $14 \%$ boron trifluoride in methanol (DBH, DBH Lab. Supplies, Poole, England) was added, sealed and placed on the heating block set at $80^{\circ} \mathrm{C}$ for $10 \mathrm{~min}$. The vial was cooled then $3 \mathrm{ml}$ distilled water 
and $3 \mathrm{ml}$ hexane were added, sealed, agitated for $1 \mathrm{~min}$ and centrifuged at $1,000 \times \mathrm{g}$ for $5 \mathrm{~min}$. The succeeding steps were done following the procedure described above for fermented soybean foods.

Extraction of fatty acids in Hanwoo longissimus muscle was done following the method described by Cho et al. (2009). Briefly, $0.5 \mathrm{mg}$ of freeze-dried beef muscle was placed into a $20 \mathrm{ml}$ headspace vial with silicone-lined cap, then $2 \mathrm{ml}$ of $\mathrm{ml}$ of $14 \%$ boron trifluoride in methanol (DBH, DBH Lab. Supplies, Poole, England) and $2 \mathrm{ml}$ of $100 \%$ methanol were added and sealed with scrimp cap. The vial was placed on the heating block set at $80^{\circ} \mathrm{C}$, vortex-mixed every $5 \mathrm{~min}$ for $2 \mathrm{~h}$. Thereafter, the vial was cooled and 3 $\mathrm{ml}$ distilled water and $3 \mathrm{ml}$ hexane were added, agitated for $30 \mathrm{~s}$ and sealed. After centrifugation at $1,000 \times \mathrm{g}$ for $5 \mathrm{~min}$, $1.0 \mathrm{ml}$ of upper layer was transferred to GLC auto-sampler vials. All samples were done in triplicates.

Fatty acids in Doenjang and Chungukjang, sesame oil and Hanwoo beef were analyzed using Gas chromatography Agilent Technology 6890N Net work GC system equipped with Flame Ionization Detector (GC-FID). GC separation of fatty acids was carried out on fused silica capillary column, (30 $\mathrm{m} \times 0.25 \mathrm{~mm} \times 0.25 \mu \mathrm{m}$, supelcowax 24079) using the temperature program of $150^{\circ} \mathrm{C}$ for $2 \mathrm{~min}$, raised from $150^{\circ} \mathrm{C}$ to $230^{\circ} \mathrm{C}$ at $10^{\circ} \mathrm{C} / \mathrm{min}$ and held at $230^{\circ} \mathrm{C}$ for $20 \mathrm{~min}$. Total running time was $30 \mathrm{~min}$. The gas flow rates for Helium as the carrier gas was $45 \mathrm{psi}, \mathrm{H}_{2}, 40 \mathrm{ml} / \mathrm{min}$ and air, $450 \mathrm{ml} / \mathrm{min}$. One (1) $\mu \mathrm{l}$ of each sample was injected into the injection port using the autosampler. Individual fatty acids were expressed as percentage of the total fatty acids detected.

\section{Sodium dodecyl sulfate polyacrylamide gel electrophoresis (SDS-PAGE) \\ SDS-PAGE of protein content was performed following} the method described by Lim et al. (2010) with some modifications. One (1) $\mathrm{g}$ each of Doenjang and Chungukjang with $2 \mathrm{ml}$ protein extraction buffer $(50 \mathrm{mM}$ Tris- $\mathrm{HCl}$ at $\mathrm{pH}$ 6.8, 5.0 $\mathrm{M}$ urea, 1\% SDS, and $2 \%$ mercaptoethanol) were stirred for $1 \mathrm{~h}$. The samples were centrifuged at $10,000 \times \mathrm{g}$ for $10 \mathrm{~min}$ and the supernatants were carefully collected using a $1 \mathrm{ml}$ syringe with needle to avoid the upper fat layer from mixing. The protein extracts were mixed with sample buffer $(125 \mathrm{mM}$ Tris- $\mathrm{HCl}$ at $\mathrm{pH}$ 6.8, 5.0 M urea, 1\% SDS, 20\% glycerol, and $0.01 \%$ bromophenol blue) at a ratio of 1:3 and were then heated in a water bath set at $95^{\circ} \mathrm{C}$ for $10 \mathrm{~min}$.

Muscle sample was powdered in liquid nitrogen using a mini-grinder. $0.6 \mathrm{~g}$ powdered muscle sample was then homogenized with $1.8 \mathrm{ml}$ of Tris-EDTA buffer $(\mathrm{pH} \mathrm{8.3)}$ ) at $14,000 \mathrm{rpm}$ for 2 min using a homogenizer (Ultra, Turrax, IKA labbortechnik, Malaysia). The protein concentration in the sample was determined using a Bio-Rad protein assay and then diluted to $2 \mathrm{mg} / \mathrm{ml}$ with sample buffer $(125 \mathrm{mM}$ Tris- $\mathrm{HCl}$ at $\mathrm{pH} 6.8,4 \%$ SDS, $1 \%, 20 \%$ glycerol, and $10 \%$ MCE, and $0.02 \%$ bromophenol blue). Before loading in the gel, the protein samples were heated in a water bath set at $95^{\circ} \mathrm{C}$ for $5 \mathrm{~min}$. The protein contents in Doenjang, Chungukjang and Hanwoo muscle were separated on $12.5 \%$ separating gel with $4 \%$ stacking gel at 200 volts until dye front runs off the end of gel. The gels were then stained by silver staining method.

\section{Analysis of flavor volatile compounds by solid phase micro-extraction and gas chromatography-mass spectrometry (SPME-GC/MS)}

Longissimus muscle tissue was powdered in liquid nitrogen using a mini-grinder. One (1) gram of the powdered beef sample, Doenjang, Chungukjang and $1 \mathrm{ml}$ sesame oil were each placed in $40 \mathrm{ml}$ headspace vial then sealed with PTFE-faced silicone septum (Supelco Co., PA, USA). The vials containing the samples were heated for 30 min in an autoclave set at $132^{\circ} \mathrm{C}$. The aroma compounds in the heated samples were analyzed using solid phase microextraction combined with gas chromatography and mass spectrometry (SPME-GS/MS) following the method described by $\mathrm{Ba}$ et al. (2010) with some modifications. After equilibration at $60^{\circ} \mathrm{C}$ for $10 \mathrm{~min}$, the heated sample vial was placed into the $60^{\circ} \mathrm{C}$ water bath, a SPME needle (Carboxen/PDMS, $75 \mu \mathrm{m}$, Supelco Co., PA, USA) was inserted into the PTFE/silicone septum and the fiber was exposed for $60 \mathrm{~min}$. The heated mixture vial was spiked with $1 \mu \mathrm{l}$ of an internal standard solution (2-methyl-3heptanone, $0.408 \mathrm{mg} / \mathrm{ml}$ in methanol) and an octagonal magnetic stirring bar also was put into the vial. At the end of extraction, the fiber was retracted, removed from the sample vial and immediately inserted into the GC injection port at $250^{\circ} \mathrm{C}$ for $5 \mathrm{~min}$. The SPME fiber was thermally conditioned at $250^{\circ} \mathrm{C}$ for $15 \mathrm{~min}$ before use by inserting it into the GC injection port to insure that there is no contamination. A gas chromatograph (Agilent Technologies 6890N Network GC System) coupled to a mass spectrometer (Agilent Technologies 5973 Network Mass Selective Detector) were used for all analyses. The SPME $75 \mu \mathrm{m}$ Carboxen/PDMS fiber (Supelco, Bellefone, PA, USA) was desorbed at $250^{\circ} \mathrm{C}$ by split mode for $5 \mathrm{~min}$ at a split ratio of 10:1 and split flow of $10 \mathrm{ml} / \mathrm{min}$. A DB-5MS capillary column, $30 \mathrm{~m} \times 0.25 \mathrm{~mm}$ i.d. $\times 0.25 \mu \mathrm{m}$ film thickness, Agilent J \& W GC columns Model No. 122-5532 (J \& W Scientific, Folcom, CA, USA) was used to separate the compounds. Helium was the carrier gas in constant pressure mode of $7.03 \mathrm{psi}$, flow rate of $1.0 \mathrm{ml} / \mathrm{min}$ and average linear velocity of $36 \mathrm{~cm} / \mathrm{s}$. The $\mathrm{GC}$ oven was programmed to $40^{\circ} \mathrm{C}$ with $5 \mathrm{~min}$ holding time, increased to $250^{\circ} \mathrm{C}$ at a rate of $8^{\circ} \mathrm{C} / \mathrm{min}$, reaching $250^{\circ} \mathrm{C}$ at $31.25 \mathrm{~min}$. 
Holding time was 5 min thus total running time was 36.25 min. MSD conditions were set as follows: capillary direct interface temperature at $250^{\circ} \mathrm{C}$; scanning mass range, 35$300 \mathrm{amu}$; EM volts, $200 \mathrm{~V}$; scan rate, $2.57 \mathrm{scan} / \mathrm{s}$. The area of each peak was integrated using the ChemStation software (Agilent Technologies, Version D.01.00, USA). A series of n-Alkanes $\left(\mathrm{C}_{8}-\mathrm{C}_{20}\right)$ were analyzed under the same conditions to obtain the linear retention index (LRI) values for the aroma volatile compounds. Authentic compounds of choice were also run under the same conditions to get the retention times for the identification of volatile components in the reaction mixtures. Each food sample was run in triplicates. The identification of volatile compounds was performed by first comparing the mass spectra with those already present in the Wiley Registry of Mass Spectral Data 7th edition (McLafferty, 2000); Agilent part no. G1035B. The second round of identification was done by comparing the linear retention index values (LRI) calculated from the standard alkane retention times with the published values in previous literatures on the website, http://www.pherobase. com/database/kovats/kovats-index.php. Identities of the compounds were further confirmed by running various authentic standards in the GC-MS. The approximate quantities of the volatile compounds were determined by comparising their peak areas with that of the 2-methyl-3heptanone internal standard obtained from the total ion chromatogram using a response factor of 1 .

\section{Statistical analysis}

Least square means and variations were calculated by a general linear model. Differences among significant means were examined with random sample error term by the Duncan's multiple test (SAS, 2007).

\section{RESULTS AND DISCUSSION}

The volatile flavor components detected in the various food types are shown in Table 1. A total of 68, 60, 49 and 50 volatile flavor compounds corresponding to 9.94, 15.75, 107.61 and $7.20 \mu \mathrm{g}$ were detected and quantified in each gram or ml of Doenjang, Chungukjang, sesame oil and cooked Hanwoo beef, respectively. Sesame oil contained significantly $(\mathrm{p}<0.05)$ the highest amount of volatile flavor compounds compared to Chungukjang, Doenjang and Hanwoo beef. According to their chemical nature, these volatile flavor components were also grouped into 10 chemical classes that include aldehydes, alcohols, acids and esters, pyrazines, ketones, hydrocarbons, furans, thiazoles, pyrroles and pyridines, and sulfur-containing compounds. The detection limit of these components in the present analytic condition was $0.012 \mu \mathrm{g} / \mathrm{g}$ of sample.

Olfactory analysis revealed that aldehydes possess some pleasant flavor such as the green floral, sweet, fruity, fatty, roast meat and almond odor notes (Specht and Baltes, 1994; Braggins, 1996; Xie et al., 2008). The numbers and amounts of aldehyes varied among food types with 19 (3.5 $\mu \mathrm{g}), 17(3.93 \mu \mathrm{g}), 6(3.25 \mu \mathrm{g})$ and $22(5.83 \mu \mathrm{g})$ aldehydes for each gram of Doenjang, Chungukjang, sesame oil and Hanwoo beef samples, respectively (Table 1). The Hanwoo longissimus tissue had significantly $(\mathrm{p}<0.05)$ higher number and total amount of aldehydes than other food types. On the other hand, the aldehydes in Doenjang, Chunjukjang and sesame oil did not differ significantly among each other. The aldehydes identified in foods in previous studies include acetaldehyde (fruity odor), 2-methylpropanal and 3-methylbutanal (green and pungent odor), and 2-methylbutanal (sweet and roasty odor) (Specht and Baltes, 1994; Huang and Ho, 2001). In the present study, these compounds were all found in the beef samples while only 3-methylbutanal and 2-methylbutanal were consistently found in Doenjang, Chungukjang and sesame oil samples. The four aldehydes are reportedly formed via the Strecker degradation of amino acids. Acetaldehyde, 2-methylpropanal, 3-methylbutanal and 2-methylbutanal are derived from alanine, valine, leucine and isoleucine, respectively (Huang and Ho, 2001). Our unpublished data similarly confirmed the generation of the flavor components by heating individual amino acids. It was particularly notable that the amounts of 3-methylbutanal was consistently higher in beef and Doenjang samples than others. The sesame oil contains higher amount of 2-methylbutanal when compared other food tested. The results are likely to some extent related to higher levels of protein degradation during ageing and ripening thereby, leading to the release of more free peptides and amino acids. It was evident that more protein bands were found in Doenjang and beef samples than in Chungukjang. The 2-methylbutanal with sweet and roasty odor notes had higher concentration in sesame oil samples than others. This is probably due to the higher roasting temperature of sesame seeds that led to generation of more 2-methylbutanal.

Aside from the four aldehydes mentioned above, most of the remaining aldehydes were derived from the fatty acid degradations in foods. Heating breaks down unsaturated fatty acids at the double bond positions and spontaneously generates intermediate hydroperoxide. Reaction intermediates of the fatty acid consequently decompose to an aldehyde and/or a radical which could be further oxidized, dehydrated and carboxylated to form aldehyde molecules (Grosch, 1982). Among the fatty acid-derived aldehydes, seven compounds including heptanal, octanal nonanal, (E)-2-decenal, decanal, 2-undecenal and tetradecanal are the major products of oleic acid (C18:1n-9) (Bading, 1970; Ba et al., 2010). The oleic acid-derived aldehydes have been reported to possess the pleasant flavors such as sweet, fatty, green, roasty, and fruity odor notes 
Table 1. Approximate quantities ( $\mu \mathrm{g} / \mathrm{g}$ or $\mathrm{ml}$ ) of volatile flavor components produced from various food types including Doenjang, Chungukjang, Sesame oil and Hanwoo beef $(n=3)$

\begin{tabular}{|c|c|c|c|c|c|c|c|}
\hline Compounds & $\mathrm{LRI}^{1}$ & Doenjang & Chungukjang & Sesame & Beef & I method $^{2}$ & F.value \\
\hline \multicolumn{8}{|l|}{ Aldehydes } \\
\hline Acetaldehyde & $\leq 800$ & - & - & - & $0.077 \pm 0.00$ & $\mathrm{Ms}+\mathrm{AC}+\mathrm{RIL}$ & \\
\hline 2-methylpropanal & $\leq 800$ & - & - & - & $0.025 \pm 0.00$ & $\mathrm{Ms}+\mathrm{AC}+\mathrm{RIL}$ & \\
\hline 3-methylbutanal & $\leq 800$ & $0.193 \pm 0.08$ & $0.114 \pm 0.03$ & $0.095 \pm 0.08$ & $0.199 \pm 0.08$ & $\mathrm{Ms}+\mathrm{AC}+\mathrm{RIL}$ & \\
\hline 2-methylbutanal & $\leq 800$ & $0.109 \pm 0.03$ & $0.063 \pm 0.01$ & $0.313 \pm 0.07$ & $0.108 \pm 0.04$ & $\mathrm{Ms}+\mathrm{AC}+\mathrm{RIL}$ & \\
\hline Pentanal & $\leq 800$ & $0.014 \pm 0.0$ & $0.051 \pm 0.01$ & - & $0.167 \pm 0.04$ & $\mathrm{Ms}+\mathrm{AC}+\mathrm{RIL}$ & \\
\hline (E),2-methyl-2-butenal & $\leq 800$ & $0.036 \pm 0.00$ & - & - & - & MS & \\
\hline Hexanal & 802 & $0.143 \pm 0.03$ & $0.203 \pm 0.06$ & $0.254 \pm 0.00$ & $0.903 \pm 0.11$ & $\mathrm{Ms}+\mathrm{AC}+\mathrm{RIL}$ & \\
\hline Fufural & 856 & $0.527 \pm 0.07$ & $0.078 \pm 0.05$ & $1.545 \pm 0.52$ & $0.023 \pm 0.00$ & $\mathrm{Ms}+\mathrm{AC}+\mathrm{RIL}$ & \\
\hline Heptanal & 905 & $0.073 \pm 0.06$ & $0.033 \pm 0.00$ & - & $0.631 \pm 0.11$ & $\mathrm{Ms}+\mathrm{AC}+\mathrm{RIL}$ & \\
\hline Methional & 931 & $0.141 \pm 0.03$ & $0.047 \pm 0.01$ & - & $0.08 \pm 0.01$ & $\mathrm{MS}+\mathrm{AC}$ & \\
\hline (E),2-heptenal & 1,070 & $0.025 \pm 0.01$ & $0.027 \pm 0.00$ & - & $0.09 \pm 0.04$ & $\mathrm{Ms}+\mathrm{AC}$ & \\
\hline 5-methyl-2-fufural & 1,104 & $0.092 \pm 0.05$ & - & - & - & MS & \\
\hline Benzaldehyde & 1,147 & $1.09 \pm 0.30$ & $2.389 \pm 0.13$ & $0.698 \pm 0.12$ & $1.367 \pm 0.3$ & $\mathrm{MS}+\mathrm{AC}$ & \\
\hline Octanal & 1,027 & - & - & - & $0.666 \pm 0.05$ & $\mathrm{Ms}+\mathrm{AC}$ & \\
\hline Benzeneacetaldehyde & 1,118 & $0.43 \pm 0.07$ & $0.382 \pm 0.13$ & - & $0.183 \pm 0.05$ & $\mathrm{Ms}+\mathrm{AC}$ & \\
\hline (E),2-octenal & 1,189 & - & $0.065 \pm 0.03$ & - & $0.124 \pm 0.02$ & $\mathrm{MS}+\mathrm{AC}$ & \\
\hline Nonanal & 1,112 & $0.268 \pm 0.12$ & $0.179 \pm 0.05$ & - & $0.862 \pm 0.15$ & $\mathrm{Ms}+\mathrm{AC}+\mathrm{RIL}$ & \\
\hline (E),2-nonenal & 1,571 & - & - & - & $0.09 \pm 0.02$ & $\mathrm{MS}+\mathrm{AC}$ & \\
\hline Decanal & 1,214 & $0.02 \pm 0.00$ & $0.04 \pm 0.01$ & - & $0.026 \pm 0.00$ & $\mathrm{MS}+\mathrm{AC}$ & \\
\hline (E)-2-decenal & 1,244 & $0.017 \pm 0.00$ & $0.054 \pm 0.00$ & - & $0.103 \pm 0.03$ & $\mathrm{MS}+\mathrm{AC}$ & \\
\hline Bezeneacetaldehyde alpha & 1,674 & $0.176 \pm 0.04$ & $0.08 \pm 0.04$ & $0.354 \pm 0.05$ & $0.023 \pm 0.00$ & MS & \\
\hline (E,E), 2,4-decadienal & 1,341 & $0.063 \pm 0.00$ & $0.064 \pm 0.01$ & - & $0.03 \pm 0.01$ & $\mathrm{MS}+\mathrm{AC}+\mathrm{RIL}$ & \\
\hline 2-undecenal & 1,356 & - & - & - & $0.055 \pm 0.02$ & $\mathrm{MS}+\mathrm{AC}$ & \\
\hline 5-methyl-2-phenyl-2-hexenal & 1,482 & $0.037 \pm 0.02$ & - & - & - & MS & \\
\hline Tetradecanal & 1,723 & $0.069 \pm 0.03$ & $0.068 \pm 0.03$ & - & $0.053 \pm 0.00$ & $\mathrm{MS}+\mathrm{AC}+\mathrm{RIL}$ & \\
\hline Total aldehydes & & $3.50^{\mathrm{b}}$ & $3.93^{\mathrm{b}}$ & $3.25^{\mathrm{b}}$ & $5.83^{\mathrm{a}}$ & & $8,341 * * *$ \\
\hline \multicolumn{8}{|l|}{ Alcohols } \\
\hline Ethanol & $\leq 800$ & $0.275 \pm 0.18$ & $0.067 \pm 0.00$ & - & - & $\mathrm{Ms}+\mathrm{AC}+\mathrm{RIL}$ & \\
\hline 1-propanol & $\leq 800$ & $0.022 \pm 0.00$ & - & - & - & $\mathrm{Ms}+\mathrm{AC}+\mathrm{RIL}$ & \\
\hline 3-methyl-1-butanol & $\leq 800$ & $0.028 \pm 0.00$ & $0.112 \pm 0.01$ & - & - & MS+RIL & \\
\hline 2-methyl-1-butanol & $\leq 800$ & $0.018 \pm 0.00$ & $0.04 \pm 0.00$ & - & - & MS+RIL & \\
\hline 2-furanmethanol & 935 & $0.215 \pm 0.03$ & - & $4.435 \pm 0.75$ & $0.042 \pm 0.01$ & $\mathrm{MS}+\mathrm{AC}$ & \\
\hline 1-hexanol & 1,328 & - & - & & $0.057 \pm 0.02$ & $\mathrm{MS}+\mathrm{AC}$ & \\
\hline 2-methylphenol & 926 & - & - & $7.676 \pm 1.63$ & - & MS & \\
\hline Phenol & 1,447 & $0.272 \pm 0.09$ & $0.221 \pm 0.02$ & $0.499 \pm 0.19$ & - & MS & \\
\hline 1-octen-3-ol & 1,085 & $0.2 \pm 0.05$ & $0.16 \pm 0.02$ & - & $0.223 \pm 0.03$ & $\mathrm{MS}+\mathrm{AC}$ & \\
\hline 1-octanol & 1,322 & $0.063 \pm 0.03$ & $0.065 \pm 0.01$ & - & - & $\mathrm{MS}+\mathrm{AC}$ & \\
\hline 2-methoxyphenol & 1,977 & $0.117 \pm 0.00$ & $0.322 \pm 0.07$ & $5.954 \pm 2.42$ & - & MS & \\
\hline 2-nonanol & 1,106 & $0.052 \pm 0.00$ & $0.047 \pm 0.01$ & - & - & $\mathrm{MS}+\mathrm{AC}$ & \\
\hline Maltol & 1,124 & $0.066 \pm 0.04$ & $0.206 \pm 0.12$ & - & - & MS & \\
\hline Benzeneethanol & 1,132 & $0.257 \pm 0.04$ & $0.376 \pm 0.2$ & - & - & MS & \\
\hline 4-ethylphenol & 1,137 & $0.049 \pm 0.02$ & - & - & - & MS & \\
\hline 4-vinylphenol & 1,234 & $0.29 \pm 0.01$ & - & - & - & MS & \\
\hline Indol & 1,311 & $0.046 \pm 0.03$ & $0.055 \pm 0.01$ & $0.169 \pm 0.01$ & - & MS & \\
\hline 2,6-dimethoxyphenol & 1,438 & $0.019 \pm 0.00$ & - & - & - & MS & \\
\hline 4-vinyl-2-methoxyphenol & 1,311 & $0.174 \pm 0.09$ & $0.161 \pm 0.01$ & $0.41 \pm 0.06$ & - & MS & \\
\hline 2-methoxy-4-vinylphenol & 1,332 & $0.20 \pm 0.07$ & - & $0.967 \pm 0.04$ & - & MS & \\
\hline 3,4-methylenedioxyphenol & 1,339 & - & - & $1.319 \pm 0.39$ & - & MS & \\
\hline Total alcohols & & $2.16^{\mathrm{b}}$ & $1.78^{\mathrm{b}}$ & $20.11^{\mathrm{a}}$ & $0.322^{\mathrm{c}}$ & & $5.32 *$ \\
\hline
\end{tabular}


Table 1. Approximate quantities $(\mu \mathrm{g} / \mathrm{g}$ or $\mathrm{ml})$ of volatile flavor components produced from various food types including Doenjang, Chungukjang, Sesame oil and Hanwoo beef $(n=3)$ (Continued)

\begin{tabular}{|c|c|c|c|c|c|c|c|}
\hline Compounds & $\mathrm{LRI}^{1}$ & Doenjang & Chungukjang & Sesame & Beef & I method $^{2}$ & F.value \\
\hline \multicolumn{8}{|l|}{ Acids and esters } \\
\hline Acetic acid & $\leq 800$ & $0.56 \pm 0.12$ & $0.127 \pm 0.01$ & $3.108 \pm 0.8$ & - & $\mathrm{MS}+\mathrm{AC}$ & \\
\hline Acetic acid, ethyl ester & $\leq 800$ & $0.305 \pm 0.00$ & $0.034 \pm 0.00$ & - & - & MS+RIL & \\
\hline Propanic acid-2-methyl,ethyl ester & $\leq 800$ & $0.084 \pm 0.00$ & $0.176 \pm 0.1$ & - & - & MS+RIL & \\
\hline 3-methylbutanoic acid & 902 & $0.202 \pm 0.14$ & $0.531 \pm 0.33$ & - & - & MS+RIL & \\
\hline 2-methylbutanoic acid & 970 & $0.132 \pm 0.1$ & $0.27 \pm 0.150$ & - & - & MS & \\
\hline Hexanoic acid ethyl, ester & 1,001 & $0.073 \pm 0.00$ & - & - & - & MS & \\
\hline Benzoic acid,methyl ester & 1,556 & $0.159 \pm 0.12$ & $0.216 \pm 0.01$ & - & - & MS & \\
\hline Benzeneacetic acid ethyl,ester & 1,244 & $0.056 \pm 0.02$ & - & - & - & MS & \\
\hline Total acids and esters & & $1.57^{\mathrm{b}}$ & $1.35^{\mathrm{b}}$ & $3.10^{\mathrm{a}}$ & 0.00 & & $9,832 * * *$ \\
\hline \multicolumn{8}{|l|}{ Pyrazines } \\
\hline Pyrazine & $\leq 800$ & - & - & $1.053 \pm 0.27$ & $0.075 \pm 0.01$ & $\mathrm{MS}+\mathrm{AC}$ & \\
\hline Methylpyrazine & 841 & $0.042 \pm 0.01$ & $0.268 \pm 0.25$ & $19.252 \pm 3.9$ & $0.05 \pm 0.00$ & $\mathrm{MS}+\mathrm{AC}+\mathrm{RIL}$ & \\
\hline 2,5-dimethylpyrazine & 922 & $1.163 \pm 0.06$ & $2.648 \pm 0.58$ & $18.602 \pm 4.09$ & $0.108 \pm 0.03$ & $\mathrm{MS}+\mathrm{AC}+\mathrm{RIL}$ & \\
\hline 2,3-dimethylpyrazine & 931 & $0.089 \pm 0.00$ & $0.809 \pm 0.54$ & - & - & MS & \\
\hline 2-vinylpyrazine & 960 & - & - & $0.684 \pm 0.19$ & - & MS & \\
\hline 2-ethyl-6-methylpyrazine & 1,004 & - & $0.067 \pm 0.04$ & $2.993 \pm 0.67$ & - & MS & \\
\hline 2-ethyl-5-methylpyrazine & 1,008 & - & - & $9.05 \pm 1.85$ & - & MS & \\
\hline Trimethylpyrazine & 1,009 & $0.288 \pm 0.02$ & $2.286 \pm 0.83$ & - & - & MS & \\
\hline Acetylpyrazine & 1,043 & $0.044 \pm 0.00$ & - & $4.032 \pm 1.02$ & - & MS & \\
\hline 2-ethyl-2,5-dimethylpyrazine & 1,526 & - & - & $4.96 \pm 1.1$ & $0.052 \pm 0.00$ & MS & \\
\hline Tetramethylpyrazine & 1,134 & $0.0466 \pm 0.4$ & $1.406 \pm 0.115$ & - & - & MS & \\
\hline 1-(6-methyl-2-pyrazinyl)-1-ethanone & 1,129 & - & - & $1.967 \pm 0.54$ & - & MS & \\
\hline 3,5-diethyl-2-methylpyrazine & 1,158 & - & - & $0.465 \pm 0.13$ & - & MS & \\
\hline 2,3,5-trimethyl-6-ethylpyrazine & 1,116 & $0.075 \pm 0.03$ & $0.083 \pm 0.05$ & - & - & MS & \\
\hline 2-methyl-5-propenylpyrazine & 1,208 & - & - & $1.217 \pm 0.33$ & - & MS & \\
\hline Total pyrazines & & $1.74^{\mathrm{c}}$ & $7.56^{\mathrm{b}}$ & $64.27^{\mathrm{a}}$ & $0.28^{\mathrm{d}}$ & & $2.2 \mathrm{E} 8 * * *$ \\
\hline \multicolumn{8}{|l|}{ Ketones } \\
\hline Acetone & $\leq 800$ & - & $0.017 \pm 0.00$ & - & - & $\mathrm{MS}+\mathrm{AC}$ & \\
\hline 2-propanone & $\leq 800$ & - & - & $0.355 \pm 0.05$ & $0.038 \pm 0.00$ & $\mathrm{MS}+\mathrm{AC}$ & \\
\hline 2,3-butanedione & $\leq 800$ & - & - & - & $0.09 \pm 0.00$ & $\mathrm{MS}+\mathrm{AC}$ & \\
\hline 2-butanone & $\leq 800$ & - & - & - & $0.076 \pm 0.01$ & $\mathrm{MS}+\mathrm{AC}$ & \\
\hline 3-hydroxy-2-butanone & $\leq 800$ & - & $0.054 \pm 0.16$ & - & $0.022 \pm 0.01$ & MS & \\
\hline 2-heptanone & 889 & $0.049 \pm 0.00$ & $0.066 \pm 0.02$ & - & $0.017 \pm 0.01$ & $\mathrm{MS}+\mathrm{AC}$ & \\
\hline 3-octanone & 1,766 & $0.232 \pm 0.03$ & $0.266 \pm 0.151$ & - & - & $\mathrm{MS}+\mathrm{AC}$ & \\
\hline Hydroxydimethylfuranone & 1,198 & - & - & $0.378 \pm 0.3$ & - & MS & \\
\hline Acetophenone & 1,341 & - & - & $1.786 \pm 0.62$ & - & MS & \\
\hline Total ketones & & $0.28^{\mathrm{b}}$ & $0.40^{\mathrm{b}}$ & $2.51^{\mathrm{a}}$ & $0.24^{\mathrm{b}}$ & & $12.99 * *$ \\
\hline \multicolumn{8}{|l|}{ Hydrocarbons } \\
\hline Toluene & $\leq 800$ & - & $0.069 \pm 0.02$ & - & $0.095 \pm 0.02$ & $\mathrm{MS}+\mathrm{AC}+\mathrm{RIL}$ & \\
\hline Styrene & 875 & $0.137 \pm 0.06$ & $0.096 \pm 0.00$ & $0.334 \pm 0.11$ & $0.034 \pm 0.00$ & MS & \\
\hline 3-ethyl-2-methyl-1,3-hexadiene & 1,069 & $0.022 \pm 0.00$ & - & - & $0.05 \pm 0.00$ & MS & \\
\hline Undecane & 1,106 & $0.044 \pm 0.01$ & - & - & $0.034 \pm 0.00$ & MS+RIL & \\
\hline Dodecane & 1,205 & $0.045 \pm 0.00$ & $0.036 \pm 0.00$ & - & $0.021 \pm 0.00$ & MS+RIL & \\
\hline Tridecane & 1,306 & $0.03 \pm 0.01$ & $0.053 \pm 0.01$ & $0.181 \pm 0.07$ & $0.016 \pm 0.00$ & MS+RIL & \\
\hline Tetradecane & 1,406 & $0.039 \pm 0.01$ & $0.048 \pm 0.01$ & - & $0.012 \pm 0.00$ & MS+RIL & \\
\hline Pentadecane & 1,507 & $0.022 \pm 0.01$ & $0.025 \pm 0.00$ & - & $0.021 \pm 0.00$ & MS+RIL & \\
\hline Total hydrocarbons & & 0.33 & 0.32 & 0.51 & 0.28 & & 4.42 \\
\hline
\end{tabular}


Table 1. Approximate quantities $(\mu \mathrm{g} / \mathrm{g}$ or $\mathrm{ml}$ ) of volatile flavor components produced from various food types including Doenjang, Chungukjang, Sesame oil and Hanwoo beef $(\mathrm{n}=3)$ (Continued)

\begin{tabular}{|c|c|c|c|c|c|c|c|}
\hline Compounds & $\mathrm{LRI}^{1}$ & Doenjang & Chungukjang & Sesame & Beef & I method $^{2}$ & F.value \\
\hline \multicolumn{8}{|l|}{ Furans } \\
\hline 1-(2-furanyl)ethanone & 915 & - & - & $1.163 \pm 0.22$ & - & MS & \\
\hline 2-butylfuran & 906 & - & - & - & $0.007 \pm 0.00$ & MS & \\
\hline 5-methylfuran & 942 & - & - & $2.196 \pm 0.31$ & - & MS & \\
\hline 2-pentylfuran & 976 & $0.221 \pm 0.00$ & $0.311 \pm 0.04$ & $0.774 \pm 0.1$ & $0.480 \pm 0.24$ & $\mathrm{MS}+\mathrm{AC}+\mathrm{RIL}$ & \\
\hline 2-hexylfuran & 1,112 & - & - & - & $0.020 \pm 0.00$ & MS & \\
\hline 2-heptylfuran & 1,140 & $0.059 \pm 0.01$ & $0.018 \pm 0.00$ & - & $0.019 \pm 0.00$ & MS & \\
\hline 2,3-dihydrobenzofuran & 1,236 & - & $0.024 \pm 0.00$ & - & - & MS & \\
\hline 2-octylfuran & 1,267 & $0.018 \pm 0.0$ & $0.029 \pm 0.00$ & - & $0.079 \pm 0.00$ & MS+RIL & \\
\hline Total furans & & $0.29^{c}$ & $0.38^{\mathrm{c}}$ & $4.13^{\mathrm{a}}$ & $0.60^{\mathrm{b}}$ & & $3.46^{*}$ \\
\hline \multicolumn{8}{|l|}{ Thiazoles } \\
\hline 2-methylthiazole & 810 & - & - & $0.145 \pm 0.06$ & - & $\mathrm{MS}+\mathrm{AC}$ & \\
\hline 4-methylthiazole & 825 & - & - & $0.965 \pm 0.29$ & - & MS & \\
\hline 5-methylthiazole & 911 & - & - & $0.248 \pm 0.05$ & - & MS & \\
\hline 2,4-dimethylthiazole & 846 & - & - & $0.869 \pm 0.36$ & - & MS & \\
\hline 2-acetylthiazole & 1,037 & $0.029 \pm 0.00$ & $0.021 \pm 0.00$ & - & $0.025 \pm 0.00$ & MS+RIL & \\
\hline 4,5-dimethylthiazole & 1,111 & - & - & $1.776 \pm 0.09$ & - & MS & \\
\hline Total thiazoles & & $0.029^{\mathrm{b}}$ & $0.021^{\mathrm{b}}$ & $4.00^{\mathrm{a}}$ & $0.025^{\mathrm{b}}$ & & $8.6^{* *}$ \\
\hline \multicolumn{8}{|l|}{ Pyrroles and pyridines } \\
\hline 1H-pyrrole & $\leq 800$ & - & - & $0.992 \pm 0.37$ & - & MS & \\
\hline $\begin{array}{l}\text { 1-methyl-5-hydroxymethyl-1,3- } \\
\text { midazole }\end{array}$ & 1,029 & - & - & $0.744 \pm 0.18$ & - & MS & \\
\hline 1H-pyrrole-2-carboxaldehyde & 1,023 & - & - & $2.619 \pm 0.09$ & - & MS & \\
\hline 2-formylpyrrole & 1,023 & $0.048 \pm 0.0$ & $0.038 \pm 0.00$ & - & - & MS & \\
\hline 3,5-dimethylisoxazole & 1,086 & - & - & $2.284 \pm 0.07$ & - & MS & \\
\hline 1-(2-pyridinyl)ethanone & 1,244 & - & - & $1.721 \pm 0.58$ & - & MS & \\
\hline 2-propylpyridine & 1,207 & - & - & $0.394 \pm 0.02$ & - & MS & \\
\hline Total pyrroles and pyridines & & $0.048^{\mathrm{b}}$ & $0.038^{\mathrm{c}}$ & $8.36^{\mathrm{a}}$ & 0.00 & & $4.06^{*}$ \\
\hline \multicolumn{8}{|l|}{ Sulfur-containing compounds } \\
\hline Methanethiol & $\leq 800$ & - & - & $0.123 \pm 0.02$ & - & MS & \\
\hline Carbondisulfide & $\leq 800$ & - & - & $0.189 \pm 0.05$ & - & MS & \\
\hline Dimethyldisulfide & $\leq 800$ & $0.076 \pm 0.00$ & $0.049 \pm 0.03$ & $0.45 \pm 0.09$ & $0.056 \pm 0.02$ & $\mathrm{MS}+\mathrm{AC}+\mathrm{RIL}$ & \\
\hline Dimethyltrisulfide & 1,296 & $0.185 \pm 0.00$ & $0.272 \pm 0.06$ & - & $0.195 \pm 0.03$ & MS & \\
\hline Fufurylmethyldisulfide & 1,239 & - & - & $0.717 \pm 0.32$ & - & MS & \\
\hline Total sulfur compounds & & $0.26^{\mathrm{b}}$ & $0.32^{\mathrm{b}}$ & $1.47^{\mathrm{a}}$ & $0.25^{\mathrm{b}}$ & & 1.07 \\
\hline Total amount/gram of sample & & $9.94^{\mathrm{c}}$ & $15.75^{\mathrm{b}}$ & $107.61^{\mathrm{a}}$ & $7.20^{\mathrm{c}}$ & & $1.9 \mathrm{E} 8 * * *$ \\
\hline
\end{tabular}

${ }^{1}$ Linear retention index calculated by applying a series of $n$-Alkanes $\left(\mathrm{C}_{8}-\mathrm{C}_{20}\right)$ using fused silica column (BD-5MS).

${ }^{2}$ Identification method: MS: the compound identified based on mass spectrum that agrees with the standard spectra in the Wiley Registry of Mass Spectral Database 7th edition; AC, the compound identified using authentic compounds; RIL, the compounds identified in agreement with linear retention index values from previous literatures using DB-5 column published on the website: http://www.pherobase.com/database/kovats/kovatsindex.php.

(-) not fund; \pm standard deviation; $* * *$ and $* * *$ significant differences at the $0.05,0.01$ and 0.001 probability levels, respectively.

${ }^{a b c}$ Mean in the same row with different superscripts differed significantly $(\mathrm{p}<0.05)$.

(Specht and Baltes, 1994). In the current study, all these compounds were present in the cooked beef at higher concentrations, but only four of them (heptanal, nonanal, E,2-decenal, tetradecanal) were found in the both Doenjang and Chungukjang samples at lower concentrations. On the other hand, sesame oil samples did not generate oleic acid- derived aldehydes, although sesame oil contained relatively higher level of oleic acids (37.8\%, Table 2). These results could be attributed to the highly significant oleic content (45.47\%) of beef thus higher amount of aldehydes were generated during heat treatment (Table 2).

Seven compounds that include pentanal, hexanal, E-2 
Table 2. Relative percentage of major fatty acid profiles in various food types including Doenjang, Chungukjang, sesame oil and Hanwoo beef $(n=3)$

\begin{tabular}{|c|c|c|c|c|c|}
\hline Fatty acid/food types & Doenjang & Chugukjang & Sesame oil & Hanwoo beef & F.value \\
\hline C8:0 & $0.036 \pm 0.01$ & - & $0.003 \pm 0.0006$ & $0.013 \pm 0.001$ & $5.01 *$ \\
\hline C10:0 & $0.021 \pm 0.001$ & - & - & $0.06 \pm 0.006$ & $2,281 * *$ \\
\hline C12:0 & $0.015 \pm 0.007$ & $0.048 \pm 0.006$ & $0.001 \pm 0.0001$ & $0.083 \pm 0.009$ & $4.06^{*}$ \\
\hline $\mathrm{C} 14: 0$ & $0.13 \pm 0.002$ & $0.123 \pm 0.005$ & $0.026 \pm 0.001$ & $2.571 \pm 0.38$ & $6,154 * * *$ \\
\hline C16:0 & $13.85 \pm 0.03$ & $13.779 \pm 0.01$ & $11.718 \pm 0.02$ & $29.928 \pm 0.465$ & $60,367 * * *$ \\
\hline C16:1 & $0.283 \pm 0.01$ & $0.209 \pm 0.015$ & $0.225 \pm 0.048$ & $6.076 \pm 0.56$ & $2.5 \mathrm{E} 7 * * *$ \\
\hline C18:0 & $4.673 \pm 0.01$ & $4.247 \pm 0.003$ & $8.272 \pm 1.910$ & $10.398 \pm 1.62$ & $2.6 \mathrm{E} 7 * * *$ \\
\hline C18:1 & $30.292 \pm 0.01$ & $20.831 \pm 0.03$ & $37.843 \pm 0.22$ & $45.469 \pm 0.264$ & $3.2 \mathrm{E} 8 * * *$ \\
\hline$C: 18: 2$ & $43.27 \pm 0.01$ & $50.908 \pm 0.035$ & $40.689 \pm 1.03$ & $4.878 \pm 0.212$ & $4.8 \mathrm{E} 7 * * *$ \\
\hline C18:3 & $6.391 \pm 0.02$ & $8.993 \pm 0.012$ & $0.3 \pm 0.007$ & $0.179 \pm 0.04$ & $99,573 * * *$ \\
\hline C20:0 & $0.454 \pm 0.008$ & $0.343 \pm 0.001$ & $0.751 \pm 0.04$ & $0.445 \pm 0.03$ & $6,956 * * *$ \\
\hline $\mathrm{C} 22: 0$ & - & $0.468 \pm 0.004$ & $0.141 \pm 0.008$ & $0.065 \pm 0.02$ & $13,755 * * *$ \\
\hline $22: 1$ & - & - & - & $0.273 \pm 0.05$ & \\
\hline $\mathrm{C} 24: 0$ & - & - & $0.017 \pm 0.003$ & $0.218 \pm 0.06$ & $6,061 * * *$ \\
\hline Total saturated & 19.763 & 19.059 & 20.938 & 43.789 & $2,971 * * *$ \\
\hline Total monounsaturated & 30.576 & 21.04 & 38.068 & 51.819 & $1,852 * * *$ \\
\hline Total polyunsaturated & 49.661 & 59.901 & 40.994 & 5.057 & $1.2 \mathrm{E} 9 * * *$ \\
\hline Polyunsaturated FA/saturated FA & 2.512 & 3.142 & 1.957 & 0.115 & $5,048 * * *$ \\
\hline
\end{tabular}

(-) without data, Values are averages of three replicates $(\mathrm{n}=3)$, \pm standard deviation.

*,**, and $* * *$ Significant differences at $0.05,0.01$ and 0.001 probability levels, respectively.

heptenal, E, 2-octenal, E, 2-nonenal, E, E, 2, 4-decadienal, and benzaldehyde were reportedly formed during the thermal degradation of linoleic acid $(\mathrm{C} 18: 2 \mathrm{n}-2)$ and linolenic acid (C18:3n-3) (Elmore et al., 2002; Ba et al., 2010). In the study of $\mathrm{Ba}$ et al. (2010) each flavor component was confirmed by heating individual polyunsaturated fatty acid. The seven aldehydes were all found in beef at higher concentrations while only four among them (petanal, hexanal, benzaldehyde and E, E, 2, 4decadienal) were found in both Doenjang and Chungukjang samples at lower amounts. Only hexanal and benzaldehyde were found in sesame oil samples. These results were in contrast to the fatty acid composition of the food samples. The relative proportions of $\mathrm{C} 18: 2 \mathrm{n}-2$ and $\mathrm{C} 18: 3 \mathrm{n}-3$ in the beef were significantly lower $(\mathrm{p}<0.05)$ than in Doenjang, Chungukjang and sesame oil (Table 2). The underlying rationale is unclear, but previous studies reported that fermented soybean pates and sesame oil generate relatively lesser and lower levels of aldehydes in number and amount than other volatile classes such as ester, alcohol, pyrazine, furan and miscellaneous (Shimoda et al., 1996; Shimoda et al., 1997; Chung, 2000; Jo et al., 2011).

Aldehydes are generally formed more than other classes of flavor compounds (Elmore et al., 1999; and Elmore et al., 2004). They are the predominant flavor component of beef as formation of the Maillard products such as pyrazines and their interaction products are limited (Bredie et al., 1998; Ames et al., 2001). The present study also revealed that aldehydes were the most predominant among the detected flavor compounds in both numbers and amounts in cooked Hanwoo beef. They presumably are the principle contributors to the 'sweet', 'green', 'roasty', 'fruity' and especially 'fatty' flavor notes characteristic of Hanwoo beef with high fat content. Furthermore, it should be taken into account that variations in kinds and amounts of some individual aldehydes like furfural with caramel, roast bread odor notes (Xie et al., 2008) could probably cause the differentiations in flavor characteristics between among food types.

A total of $18,12,8$ and 3 alcohol compounds corresponding to $2.16,1.78,20.11$ and $0.322 \mu \mathrm{g}$ were detected and approximated in each gram of Doenjang, Chungukjang, sesame oil and beef, respectively (Table 1). The beef sample showed 1-hexanol and 1-octen-3-ol and 2-furanmethanol which commonly appear in cooked beef at medium concentrations (Elmore et al., 1999; Elmore et al., 2004). The 1-hexanol and 1-octen-3-ol were derived from linoleic acid (Elmore et al., 2002) while 2-furanmethanol is a product of Maillard reactions (Ames et al., 2001). The formation of alcohol compounds is triggered by thermal degradations of fatty acids (Macleod, 1994; Elmore et al., 2002), and Maillard reactions (Ames et al., 2001). In addition, fermentation and roasting processes used for the production of fermented soybean pastes and sesame oil are probably related to the formation of some alcohols. Some odor compounds that are particularly important for cooked 
foods were also identified such as 1-octen-3-ol (mushroom odor note), phenol (woody and sweet), 4-ethylphenol (roasted, smoky and burnt), 2-nonanol (fruity odor note) and 2-methoxyphenol (sweet and smoky) (Guen et al., 2000; Xie et al., 2008). It was noted more alcohol compounds were found in Doenjang than in Chungukjang. The difference in the number of alcohols between Doenjang and Chungukjang are presumably due to different processing methods but less likely related to the raw materials. In the present study, the concentration of ethanol in Doenjang was nearly 4 times greater than in Chungukjang and the other alcohol compounds present like 1-propanol. This is likely related to the fermentation of sugar where yeast converts sugar into ethanol and carbon dioxide. Maltol, 1-octen-3-ol, 2-methoxy-4-vinylphenol, ethanol and 4-ethylphenol were credited for the sensory attributes of Doenjang (Lee and Ahn, 2009; Jo et al., 2011), however, only 2-methoxy-4-vinylphenol and 4-ethylphenol were found in the current study. On the other hand, the amount of maltol in Chungukjang was nearly 3 times higher than in Doenjang. The differences in numbers and amounts alcohol compounds may account for the differences in the characteristic flavor of Doenjang and Chungukjang. Almost all the detected alcohols in the sesame oil samples were higher in concentration than the other food types. This is presumably due to the high roasting temperature for sesame seeds. Ames et al. (2001) reported that the kinds and amounts of volatile flavor components increase with heating temperature.

Three acids and 5 esters were identified in the current study (Table 1). Acetic acid, 3-methylbutanoic acid and 2-methylbutanoic acid were found in both Doenjang and Chungukjang samples but only acetic acid was present in sesame oil while none of them was found in beef. These acid compounds were also found in various fermented soybean pastes and sesame oil in previous studies (Shimoda, 1996; Lee and Ahn, 2009; Jo et al., 2011; Zhao et al., 2011). Taking the formation pathways of these acids, results are understandable. In particular, 3-methylbutanoic acid was reported to be formed from leucine amino acid catabolism via transamination (Czerny and Schieberle, 2005). The 3-methylbutanoic acid imparts sweaty and cheese-like odor notes together with acetic acid in fermented soybean foods (Shukla et al., 2010, Zhao et al., 2011) while 2 and 3-methylbutanoic acid were reported to influence the quality of Chungukjang (Choi et al., 1998).

There were five esters were found in Doenjang, three of them were also found in Chungukjang while none was detected in sesame oil and beef samples. Most of these esters have been previously identified in Doenjang samples (Lee and Ahn, 2009; Jo et al., 2011). Elmore et al. (2002) observed that many esters are formed in reaction mixtures containing amino acid, ribose and fatty methyl esters. In addition, Chou and Hwan (1994) reported that actions of fungal lipase on soybean lipids probably produce esters. Esters have been reported to have odor notes such as fruity, pineapple and honey (Bauer and Garbe, 1985) and have been recently reported to impart overall briny, soy sauce and cooked soybean flavors (Jung and Kang, 2002). The current results showed that more kinds of esters are formed in Doenjang than Chungukjang. The variations in the number and concentration of esters could be probably due to the differences in fermentation process as well as the fermenting microorganisms used. Although these esters are present in small quantities, together with volatile acids they probably cause the differentiations in flavor characteristics among the food samples.

Pyrazines are very important flavor components in cooked foods and they have been well known to produce nutty, musty, cocoa, peanut, roasty odor notes in fermented soybean pastes, sesame oil and cooked beef (Ho and Carlin, 1989; Cerny and Grosch, 1992; Setcht and Baltes, 1994; Schieberle, 1996). Pathways for pyrazines are through the Maillard reactions of sugars with amino acids (Mottram, 1994). In addition, the action of free ammonia on sugar and/or lipid oxidation products has been known to generate pyrazines (Whitfield, 1992). The current study identified 7, 7, 11 and 4 pyrazines which correspond to $1.74,7.56,64.27$ and $0.28 \mu \mathrm{g} / \mathrm{g}$ of Doenjang, Chungukjang, sesame oil and beef, respectively. The amount of pyrazine differed significantly $(\mathrm{p}<0.05)$ among food types. Pyrazines have been reported to be important contributors to the flavor of various fermented soybean paste products (Lee and Ahn, 2009; Jo et al., 2011). Doenjang and Chungukjang were observed to generate 6 similar pyrazines although the concentrations of the compounds differ between the two products. The major compounds were 2,5-dimethylpyrazine, trimethylpyrazine and, tetramethylpyrazine which were determined to be several times higher in Chungukjang. Lee and Ahn (2009) reported similar findings. The differences in $\mathrm{pH}$ and moisture contents of Doenjang and Chungukjang probably affected the formation of these pyrazines during sample preparation for the detection of flavor component by GC/MS technique. Ames et al. (2001) reported that the amounts of pyrazines increased with $\mathrm{pH}$.

Noticeably, sesame oil highest pyrazine content accounting to half the total volatile components that were detected. Methylpyrazine, 2, 5-dimethylpyrazine and 2-ethyl-5-methylpyrazine were the most predominant compounds. This is in agreement with the findings of Shimoda et al. $(1996 ; 1997)$ that pyrazines were the major volatile compound class in sesame oil and its amount accounted to $45 \%$ in amounts of the total detected flavor components. The higher amounts of pyrazines in sesame oil samples compared to other foods are likely due to the high roasting temperature of sesame seeds. It has been well 
documented that high temperature treatment increases both the participation of free amino acids/peptides to produce pyrazines during Maillard reactions and the formation of intermediate reactants such as nitrogen compounds like ammonia for the formation of pyrazines (Jung et al., 1997; Shimoda et al., 1997; Ames et al., 2001). Thus, the relatively high amounts of pyrazines together with their pleasant flavor odor notes (roasted-like, nutty) are considered as the most important contributors to the flavor characteristics of sesame oil. On the other hand, only 4 pyrazines with relatively low concentrations were observed in cooked beef samples and these compounds also commonly appeared in other food types. This is probably due to low heating temperature $\left(132^{\circ} \mathrm{C}\right)$ used in the current study which seems to be not ideal for the formation of pyrazines. Higher amounts of pyrazines associated with stronger roasted odor note are usually observed in meat cooked by roasting (Specht and Baltes, 1994). This suggeststhat higher cooking temperature is needed for the generation of pyrazine flavor notes from beef steaks.

Ketones and hydrocarbons are generally formed via the thermal degradation processes of fatty acids (Maclead, 1994). In the current study, the amounts of total ketones and hydrocarbons were higher in sesame oil than other food types. This is likely associated with higher level of oxidation of polyunsaturated fatty acids during roasting of sesame seeds. However, in terms of total amounts, this class of odor notes was very limited compared to other flavor classes.

Furans have been known to form during thermal degradation of fatty acids (Elmore et al., 2002). The compound 2-pentylfuran that originated from $\mathrm{C} 18: 3 \mathrm{n} 3$ is the common compound found in all food samples. The furans identified in the current study were previously reported in fermented soybean pastes, sesame oil and beef (Shimoda et al., 1997; Elmore et al., 1999; Jo et al., 2011). Sesame oil had the highest furan content among the food samples that were tested. This is probably due to the high polyunsaturated fatty acid content of sesame seeds and indirectly influenced by high roasting temperature that resulted in higher degree of lipid thermal degradation. Shimoda et al. (1997) found that furans account for $8.3 \%$ of the total amount of total detected flavor components in sesame oil. It was assumed that although furans have various odor notes, their odors are probably weak and/or masked by the other aroma compounds with intense odor notes (Specht and Baltes, 1994).

The kinds and amounts of thiazoles widely varied among the food samples. Sesame oil had highest level of thiazoles. Both 4-methylthiazole and 4,5-dimethylthiazole were the most abundant thiazoles in the sesame oil. The compounds have been reported to be the principle contributors of sesame oil seed flavor (Shimoda et al., 1996). On the other hand, low concentration of 2-acetylthiazole, associated with roasty and sulphury odor notes (Schieberle, 1996), was found in Doenjang, Chungukjang and beef but not in sesame oil. Shimoda et al. (1996) reported that thiazoles greatly increased with roasting temperatures of $130-180^{\circ} \mathrm{C}$. This is likely the reason why very less thiazoles were found in Doenjang, Chungukjang and beef.

Pyrroles and pyridines are the Maillard products containing nitrogenous atom in the structures. Their amounts were highest in sesame oil samples. Similar results were reported by Shimoda et al. (1997). The only compound found in the Doenjang and Chungukjang samples was 2-formylpyrrole. Sulfur compounds are formed from the Strecker degradation of cysteine or cystine during Maillard reaction. They generally possess sulfuryl, sweety and onion odor notes (Machiels et al., 2004). In the current study, dimethyldisulfide was found in all four food types, and were previously detected in various fermented soybean pastes, sesame oil and cooked beef (Shimoda et al., 1996; Ba et al., 2010; Jo et al., 2011).

\section{IMPLICATIONS}

The current data suggests that the differences in kinds and quantities of volatile flavor components between food types were largely related to fatty acids, protein contents and as well as the processing procedure such as cooking methods between Korean traditional food types. Sesame oil showed highest kinds and amounts of flavor components which beef longissimus muscle had lowest levels. Aldehydes are the most predominant flavor class in cooked beef. This is probably due to higher levels of fatty acid degradations and free amino acids/peptides in meat during ageing and on heating. On the other hand, the high aroma intense compounds like pyrazines and sulfur-containing compounds were limited in cooked beef in the current experimental condition (e.g., relatively low heating temperature). This was likely the reason why aldehydes even with low odor thresholds were not inhibited and/or masked by other high aroma intense compounds. This suggested that higher heating (or roasting) temperature is required for high aroma notes in addition to aldehydes. Furthermore, this implies that proteolytic activity and stability of fatty during ageing have a great influence on the generation of flavor components in cooked beef.

\section{ACKNOWLEDGEMENT}

It should be acknowledged that this work was partly supported by a grant from the Next-Generation BioGreen 
21 Program (No. PJ008191) and FTA issue project (No. J9070552011), Rural Development Administration, Republic of Korea.

\section{REFERENCES}

Ames, J. M., R. C. E. Guy and G. J. Kipping. 2001. Effect of pH and temperature on the formation of volatile compounds in cysteine/reducing sugar/Starch mixtures during extrusion cooking. J. Agric. Food Chem. 49:1885-1894.

Ba, H. V., M. C. Oliveros, K. S. Ryu and I. H. Hwang. 2010. Development of analysis condition and detection of volatile compounds from cooked beef by SPME-GC/MS analysis. Korean J. Food Sci. Anim. Resour. 30:73-86.

Bading, H. T. 1970. Cold - storage defects in butter and their relation to the autoxidation of unsaturated fatty acids. Med. Melk-Zuiveltijdschr. 24:147-171.

Bauer, K. and D. Garbe. 1985. Common fragrance and flavor materials preparation: properties and uses. Deerfield Beach, VCH publisher Inc.

Braggins, T. J. 1996. Effect of stress-related changes in sheep meat ultimate PH on cooked odor and flavor. Food Chem. 44:23522360.

Bredie, W. L. P., D. S. Mottram and R. C. E. Guy. 1998. Aroma volatiles generated during extrusion cooking of maize flour. J. Agric. Food Chem. 46:1479-1487.

Cerny, C. and W. Grosch. 1992. Evaluations of potent odorants in roasted beef by aroma extract dilution analysis. Zeitschrift für Lebensmitteluntersuchung und Forschung A. 194:322-325.

Cho, W. B., M. C. Oliveros, K. M. Park, K. T. Do, K. H. Lee, K. S. Seo, J. G. Choi, M. J. Lee, B. C. Choi, K. S. Ryu and I. H. Hwang. 2009. Objective and subjective quality characteristics of pork longissimus muscle as a function of the ultimate $\mathrm{pH}$. Korean J. Food Sci. Anim. Resour. 6:685-694.

Choi, U. K., D. H. Son, W. D. Ji, M. H. Im, J. D. Choi and Y. G. Chung. 1998. Changes of taste components and palatability during Chungukjang fermentation by Bacillus subtilis DC-2. J. Kr. Soc. Food Sci. Nutr. 27:840-845.

Chou, C. C. and C. H. Hwan. 1994. Effect of ethanol on the hydrolysis of protein and lipid during the ageing of Chinese fermented soya bean curds (sufu). J. Sci. Food Agric. 66:393398.

Chung, H. Y. 2000. Volatile flavor components in red fermented soybean (Glycine max) curds. J. Agric. Food Chem. 48:18031809.

Chung, J. and E. O. Choe. 2001. Effects of sesame oil on thermooxidative stability of soybean oil. Food Sci. Biotechnol. 10:446-450.

Czerny, M. and P. Schieberle. 2005. Labeling studies on pathways of odorant generation by Saccharomyces cerevisiae in wheat bread doughs. In: Proceeding of the 11th Weurman Flavor Research Symposium (Lecture No. 11), Denmark.

Elmore, J. S., D. S. Mottram, M. Enser and J. D. Wood. 1999. Effect of the polyunsaturated fatty acid composition of beef muscle on the profile of aroma volatiles. J. Agric. Food Chem. 47:1619-1625.

Elmore, J. S., H. E. Warren, D. S. Mottram, N. D. Scollan, M. Enser, R. I. Richardson and J. D. Wood. 2004. A comparison of the aroma volatiles and fatty acid compositions of grilled beef muscle from Aberdeen Angus and Holstein-Friesian steers fed diets based on silage or concentrates. Meat Sci. 68:27-33.

Elmore, J. S., M. M. Campo, M. Enser and D. S. Mottram. 2002. Effect of Lipid composition on Meat-like model systems containing cystein, ribose and polyunsaturated fatty acids. J. Agric. Food Chem. 50:1126-1132.

Grosch, W. 1982. Lipid degradation products and flavor. In: Food Flavors (Ed. I. D. Morton and A. J. MacLeod). Elsevier, Amsterdam. pp. 325-386.

Guen, S. L., C. Prost and M. Demaimay. 2000. Critical comparison of three olfactometric methods for identification of the most potent odorants in cooked mussels (Mytilus edulis). J. Agric. Food Chem. 4:1307-1314.

Ho, C. T. and J. T. Carlin. 1989. Formation and aroma characteristics of heterocyclic compounds in foods. In: Flavor chemistry, trends and development (Ed. R. Teranishi, R. G. Buttery and F. Shahidi). Washington, DC, American chemical society. pp. 92-104.

Huang, T. C. and C. T. Ho. 2001. Flavors of meat products. In: Meat Science and Application (Ed. Y. H. Hui, W. K. Nip, R. W. Roger and O. A. Young). Marcel Dekker, Inc. pp.71-102.

Hwang, Y. H., G. D. Kim, J. Y. Jeong, S. J. Hur and S. T. Joo. 2010. The relationship between muscle fiber characteristics and meat quality traits of highly marbled Hanwoo (Korean native cattle) steers. Meat Sci. 86:456-461.

Jung, M. Y., J. Y. Bock, S. O. Back, T. K. Lee and J. H. Kim. 1997. Pyrazine contents and oxidative stabilities of roasted soybean oil. Food Chem. 1:95-102.

Jung, Soon Teck? and Kang, Seong-Gook. 2002. "The Past and Present of Traditional Fermented Foods in Korea". http://www.miyajima-soy.co.jp/science/kouenkai/kouenkai.htm

Kim, C. J. and E. S. Lee. 2003. Effects of quality grade on the chemical, physical and sensory characteristics of Hanwoo (Korean native cattle) beef. Meat Sci. 63:397-405.

Kim, D. H., Y. K. Kim, Y. H. Chung, Y. M. Yoo and B. Y. Park. 1993. A study on the consumer's attitude to beef: 1 . Consumer's purchasing pattern and preference. RDA J. Agric Sci. 35:598-601.

Lee, D. S., B. S. Noh, S. Y. Bae and K. Kim. 1998. Characterization of fatty acids composition in vegetable oils by gas chromatography and chemometrics. Anal. Chim. Acta. 358:163-175.

Lee, S. J. and B. M. Ahn. 2009. Comparison of volatile components in fermented soybean pastes using simultaneous distillation and extraction (SDE) with sensory characterization. Food Chem. 114:600-609.

Lim, J. Y., J. J. Kim, D. S. Lee, G. H. Kim, J. Y. Shim, I. H. Lee and J. Y. Imm. 2010. Physicochemical characteristics and production of whole soymilk from Monascus fermented soybeans. Food Chem. 120:255-260.

Machiels, D., L. Istasse and S. M. van Ruth. 2004. Gas chromatography-olfactometry analysis of beef meat originating from differently fed Belgian Blue, Limousin and Aberdeen Angus bulls. Food Chem. 86:377-3831.

Machiels, D., S. M. van Ruth, M. A. Posthumus and L. Istasse. 2003. Gas chromatography-olfactometry analysis of the volatile compounds of two commercial Irish beef meats. Talanta 60:755-764 
MacLeod, G. 1994. The flavor of beef. In: Flavor of meat and meat products (Ed. F. Shahidi). Glasgow, Chapman and Hall. pp. 437.

Madruga, M. S. and D. S. Mottram. 1995. The effect of PH on the formation of Maillard-derived aroma volatiles using a cooked meat system. J. Sci. Food Agric. 68:305-310.

Mottram, D. S. 1994. Some aspects of the chemistry of meat flavor. In the flavor of meat and meat products (Ed. F. Shahidi). Blackie, Glasgow. pp. 210-230.

Panjono, S. M. Kang, I. S. Lee and S. K. Lee. 2009. Carcass characteristics of Hanwoo (Korean cattle) from different sex conditions, raising altitudes and slaughter seasons. Livest. Sci. 123:283-287.

Park, M. K., I. H. Cho, S. Lee, H. K. Choi, D. Y. Kwon and Y. S. Kim. 2010. Metabolite profiling of Cheongukjang, a fermented soybean paste, during fermentation by gas chromatographymass spectrometry and principal component analysis. Food Chem. 122:1313-1319.

Robbins, K., J. Jensen, K. J. Ryan, C. Homco-Ryan, F. K. McKeith and M. S. Brewer. 2003. Consumer attitudes towards beef and acceptability of enhanced beef. Meat Sci. 65:721-729.

Savell, J. W., R. E. Branson, H. R. Cross, D. M. Stiffler, J. W. Wise, D. B. Griffin and G. C. Smith. 1987. National consumer retail beef study: palatability evaluations of beef loin steaks that differed in marbling. J. Food Sci. 52:517-519.

Schieberle, P. 1996. Odor-active compounds in moderately roasted sesame. Food Chem. 2:145-152.
Shi, H. and C. T. Ho. 1994. Flavor of poultry meat. In: the flavor of meat and meat products (Ed. F. Shahidi). Blackie Glasgow pp. 52-71.

Shimoda, M., H. Shiratsuchi, Y. Nakada, Y. Wu and Y. Osajima. 1996. Identification and sensory characterization of volatile flavor compounds in sesame seed oil. J. Agric. Food Chem. 44:3909-3912.

Shimoda, M., Y. Nakada, M. Nakashima and Y. Osajima. 1997. Quantitative comparison of volatile flavor compounds in deeproasted and light-roasted sesame seed oil. J. Agric. Food Chem. 45:3193-3196.

Shukla, S., T. B. Choi, H. K. Park, M. H. Kim, I. K. Lee and J. K. Kim. 2010. Determination of non-volatile and volatile organic acids in Korean traditional fermented soybean paste (Doenjang). Food Chem. Toxicol. 48:2005-2010.

Sonntag, N. O. V. 1981. Composition and characteristics of individual fats and oils. In: Bailey's Industrial Oil and Fat Products (Ed. D. Swern, John Wiley and Sons). Inc, New York. pp. 289-477.

Whitfield, F. B. 1992. Volatiles from interactions of Maillard reactions and lipids. CRC Crit. Rev. Food Sci. Nutr. 31:1-58.

Xie, J. C., B. G. Sun and S. B. Wang. 2008. Aromatic constituents from Chinese traditional smoke-cured bacon of mini-pig. Food Sci. Technol. Int. 14:329-340.

Zhao, J. X., X. J. Dai, X. M. Liu, H. Zhang, J. Tang and W. Chen. 2011. Comparison of aroma compounds in naturally fermented and inoculated Chinese soybean pastes by GC-MS and GCOlfactometry analysis. Food Control 22:1008-1013. 